\title{
Fast Converging Series for Riemann Zeta Function
}

\author{
Hannu Olkkonen ${ }^{1}$, Juuso T. Olkkonen ${ }^{2}$ \\ ${ }^{1}$ Department of Applied Physics, University of Eastern Finland, Kuopio, Finland \\ ${ }^{2}$ VTT Technical Research Centre of Finland, Espoo, Finland \\ Email: hannu.olkkonen@uef.fi, juuso.olkkonen@vtt.fi
}

Received August 29, 2012; revised September 13, 2012; accepted September 25, 2012

\begin{abstract}
Riemann zeta function $\zeta(s)$ has a key role in number theory and in its applications. In this paper we present a new fast converging series for $\zeta(s)$. Applications of the series include the computation of the $\zeta(3)$ and recursive computation of $\zeta(2 s+1), \zeta(2 s)$ and generally $\zeta(s), s \in \mathbf{R}$. We discuss on the production of irrational number sequences e.g. for encryption coding and zeta function maps for analysis and synthesis of log-time sampled signals.
\end{abstract}

Keywords: Riemann Zeta Function; Converging Series; Number Theory; Cryptography; Signal Processing

\section{Introduction}

Riemann zeta function plays an important role in modern number theory and its applications, especially in information theory and signal processing [1]. The Riemann zeta function $\zeta(s)$ is defined for complex numbers $s$ with $R\{s\}>1$ by

$$
\zeta(s)=\sum_{n=1}^{\infty} \frac{1}{n^{s}}
$$

For $s \in \mathbf{N}$ the Riemann zeta function is of the general form

$$
\zeta(2 s)=\frac{\pi^{2 s}}{N(s)}
$$

where $N(s)$ is an integer. For example $\zeta(2)=\pi^{2} / 6$, $\zeta(4)=\pi^{4} / 90$ and $\zeta(6)=\pi^{6} / 945$.

Closed form solution for $\zeta(2 s+1), s \in \mathbf{N}$ is not known. Especially the infinite sums converging to $\zeta(3)$ have been extensively studied. Apery has proved that $\zeta(3)$ is irrational $[2,3]$. However, his proof does not concern on other values of $\zeta(2 s+1)$. Also, it is not known if $\zeta(3)$ is transcendental or not. The series (1) converges very slowly. Some acceleration on this series is achieved by asymptotic expansion with Bernoulli numbers [4]. In this context the Euler's transformation is also efficient. Integral representations of the Riemann zeta function at odd integers has been described in [5, $6]$.

In this work we describe some new results on the converging series of the zeta function. Our primary aim is to develop fast converging series for $\zeta(2 s+1), s \in \mathbf{N}$. The main result is as follows.

Theorem 1. Suppose that $\zeta(s)$ is the Riemann zeta function defined by (1). The following infinite series converges as $\sum_{s=2}^{\infty} \frac{\zeta(s)}{2^{s}}=\ln 2$.

In Section 2 we provide a proof of Theorem 1. In Section 3 we present some applications of the Theorem 1, which include the converging series for even and odd Riemann function (Lemmas 1 and 2) and fastly converging series for $\zeta(3)$ (Lemma 3). In Section 4 we apply the results of the Theorem 1 to develop fast recursive method for computing the $\zeta(s)$ values (Lemma 4). In Section 5 , we describe a modified version of the Theorem 1.

\section{Proof of Theorem 1}

We may write

$$
\begin{aligned}
\sum_{s=2}^{\infty} \frac{\zeta(s)}{2^{s}} & =\sum_{s=2}^{\infty} \frac{1}{2^{s}} \sum_{n=1}^{\infty} \frac{1}{n^{s}}=\sum_{n=1}^{\infty} \sum_{s=2}^{\infty}\left(\frac{1}{2 n}\right)^{s} \\
& =\sum_{n=1}^{\infty} \frac{1}{(2 n)^{2}} \sum_{s=0}^{\infty}\left(\frac{1}{2 n}\right)^{s} \\
& =\sum_{n=1}^{\infty} \frac{1}{2 n(2 n-1)}
\end{aligned}
$$

Then we apply the well known logarithmic series

$$
\ln 2=\sum_{n=1}^{\infty} \frac{(-1)^{n+1}}{n}=1-\frac{1}{2}+\frac{1}{3}-\frac{1}{4}+\cdots
$$

By grouping the terms into pairs we obtain 


$$
\begin{aligned}
\ln 2 & =\left(1-\frac{1}{2}\right)+\left(\frac{1}{3}-\frac{1}{4}\right)+\cdots \\
& =\sum_{n=1}^{\infty}\left(\frac{1}{2 n-1}-\frac{1}{2 n}\right)=\sum_{n=1}^{\infty} \frac{1}{2 n(2 n-1)}
\end{aligned}
$$

Due to (3) we have

$$
\ln 2=\sum_{s=2}^{\infty} \frac{\zeta(s)}{2^{s}}
$$

which completes the proof.

\section{Applications of Theorem 1}

Lemma 1.

$$
\sum_{s=1}^{\infty} \frac{\zeta(2 s)}{2^{2 s}}=\frac{1}{2}
$$

Proof: We may write

$$
\begin{aligned}
\sum_{s=1}^{\infty} \frac{\zeta(2 s)}{2^{2 s}} & =\sum_{s=1}^{\infty} \frac{1}{2^{2 s}} \sum_{n=1}^{\infty} \frac{1}{n^{2 s}} \\
& =\sum_{n=1}^{\infty} \frac{1}{4 n^{2}} \sum_{s=0}^{\infty}\left(\frac{1}{4 n^{2}}\right)^{s}=\sum_{n=1}^{\infty} \frac{1}{4 n^{2}-1}
\end{aligned}
$$

By dividing the last series into two parts we have

$$
\begin{aligned}
\sum_{n=1}^{\infty} \frac{1}{4 n^{2}-1} & =\frac{1}{2} \sum_{n=1}^{\infty}\left(\frac{1}{2 n-1}-\frac{1}{2 n+1}\right) \\
& =\frac{1}{2} \sum_{n=0}^{\infty} \frac{1}{2 n+1}-\frac{1}{2} \sum_{n=1}^{\infty} \frac{1}{2 n+1}=\frac{1}{2}
\end{aligned}
$$

Lemma 2.

$$
\sum_{s=1}^{\infty} \frac{\zeta(2 s+1)}{2^{2 s+1}}=\ln 2-\frac{1}{2}
$$

Proof: We may write

$$
\begin{aligned}
\sum_{s=1}^{\infty} \frac{\zeta(2 s+1)}{2^{2 s+1}} & =\sum_{s=1}^{\infty} \frac{1}{2^{2 s+1}} \sum_{n=1}^{\infty} \frac{1}{n^{2 s+1}}=\sum_{s=0}^{\infty}\left(\frac{1}{4 n^{2}}\right)^{s} \\
& =\sum_{n=1}^{\infty} \frac{1}{2 n\left(4 n^{2}-1\right)}
\end{aligned}
$$

By separating the last series into two parts and due to Theorem 1 and (9) we have

$$
\begin{aligned}
\sum_{n=1}^{\infty} \frac{1}{2 n\left(4 n^{2}-1\right)} & =\sum_{n=1}^{\infty} \frac{1}{2 n(2 n-1)}-\sum_{n=1}^{\infty} \frac{1}{4 n^{2}-1} \\
& =\ln 2-\frac{1}{2}
\end{aligned}
$$

Lemma 3.

$$
\zeta(3)=8 \ln 2-4-\sum_{n=1}^{\infty} \frac{1}{n^{3}\left(4 n^{2}-1\right)}
$$

Proof: We may write (10) as

$$
\begin{aligned}
\zeta(3) & =8 \ln 2-4-8 \sum_{s=2}^{\infty} \frac{\zeta(2 s+1)}{2^{2 s+1}} \\
& =8 \ln 2-4-\sum_{n=1}^{\infty} \frac{1}{n^{3}\left(4 n^{2}-1\right)}
\end{aligned}
$$

which equals (13).

\section{Recursive Computation of $\boldsymbol{\zeta}(\boldsymbol{s})$}

Lemma 4. $\zeta(s)=4 R(s-2)-R(s)$, where

$$
R(s)=\sum_{n=1}^{\infty} \frac{1}{n^{s}\left(4 n^{2}-1\right)}
$$

Proof: First we prove the Lemma 4 for $\zeta(5)$. Based on Lemma 2 we can write the first two terms as

$$
\frac{\zeta(3)}{8}+\frac{\zeta(5)}{32}+\frac{1}{32} \sum_{n=1}^{\infty} \frac{1}{n^{3}\left(4 n^{2}-1\right)}=\ln 2-\frac{1}{2}
$$
have

By substituting the equation for $\zeta(3)$ (Lemma 3) we

$$
\zeta(5)=\sum_{n=1}^{\infty} \frac{4}{n^{3}\left(4 n^{2}-1\right)}-\sum_{n=1}^{\infty} \frac{1}{n^{5}\left(4 n^{2}-1\right)}
$$

Correspondingly, for $\zeta(7)$ we obtain

$$
\zeta(7)=\sum_{n=1}^{\infty} \frac{4}{n^{5}\left(4 n^{2}-1\right)}-\sum_{n=1}^{\infty} \frac{1}{n^{7}\left(4 n^{2}-1\right)}
$$

and generally

$$
\zeta(s)=\sum_{n=1}^{\infty} \frac{4}{n^{s-2}\left(4 n^{2}-1\right)}-\sum_{n=1}^{\infty} \frac{1}{n^{s}\left(4 n^{2}-1\right)}
$$

By denoting

$$
R(s)=\sum_{n=1}^{\infty} \frac{1}{n^{s}\left(4 n^{2}-1\right)}
$$

we obtain Lemma 4.

\section{Modified Version of Theorem 1}

Let us consider the zeta series

$$
\sum_{s=2}^{\infty} \frac{\zeta(s)-1}{2^{s}}
$$

Due to the limit value $\zeta(\infty)=1$ the nominator approaches zero and the series has accelerated convergence. We directly have

$$
\sum_{s=2}^{\infty} \frac{\zeta(s)-1}{2^{s}}=\sum_{s=2}^{\infty} \frac{\zeta(s)}{2^{s}}-\sum_{s=2}^{\infty} \frac{1}{2^{s}}=\ln 2-\frac{1}{2}
$$

Due to Lemma 1 we obtain, correspondingly 


$$
\begin{aligned}
\sum_{s=1}^{\infty} \frac{\zeta(2 s)-1}{2^{2 s}} & =\sum_{s=1}^{\infty} \frac{\zeta(2 s)}{2^{2 s}}-\sum_{s=1}^{\infty} \frac{1}{2^{2 s}} \\
& =\frac{1}{2}-\frac{1}{3}=\frac{1}{6}
\end{aligned}
$$

Finally, we have (Lemma 2)

$$
\begin{aligned}
\sum_{s=1}^{\infty} \frac{\zeta(2 s+1)-1}{2^{2 s+1}} & =\sum_{s=1}^{\infty} \frac{\zeta(2 s+1)}{2^{2 s+1}}-\sum_{s=1}^{\infty} \frac{1}{2^{2 s+1}} \\
& =\ln 2-\frac{1}{2}-\frac{1}{6}=\ln 2-\frac{2}{3}
\end{aligned}
$$

\section{Discussion}

One application of the present main result (Theorem 1) is the computation of the Riemann zeta function $\zeta(s)$ for odd $s$. The series for $\zeta(3)$ (Lemma 3 ) is rapidly converging. First 120 terms of the sum yield the 9 th correct decimals. The use of Euler's transformation yields the infinite sum for $\zeta(3)$

$$
\zeta(3)=\frac{4}{3} \sum_{n=0}^{\infty} \frac{(-1)^{n}}{(n+1)^{3}}
$$

However, first 1600 terms is needed for the 9 correct decimals. Due to Lemma 4 the convergence of the series of can be accelerated by recursive computation:

$$
\begin{aligned}
& \zeta(3)=8 \ln 2-4-R(3) \\
& \zeta(5)=4 R(3)-R(3) \\
& \vdots \\
& \zeta(s)=4 R(s-2)-R(s)
\end{aligned}
$$

To obtain 9 correct decimals we need 30 terms of the summation $R(s)$ for computation of $\zeta(5), 9$ terms for $\zeta(7)$ and 7 terms for $\zeta(9)$. An interesting observation is that thought the recursive computation method was deduced for $\zeta(2 s+1)$, it is valid also for computation of the $\zeta(2 s)$ values. An alternative method to obtain the $\zeta(2 s)$ values would be the deduction of the recursive method similar to Lemma 4 based on Lemma 1. However, the convergence of the series is not so accelerated.

The modified zeta series (21 - 23) have accelerated convergence. However, their application yields the same results for the series for $\zeta(3)$ (Lemma 3 ) and the algorithm for recursive computation of $\zeta(s)$ (Lemma 4). The fast convergence of the zeta series $(21-23)$ has a significant value in testing the $\zeta(s)$ values. Previously the series $\sum_{s=2}^{\infty}[\zeta(s)-1]=1, \sum_{s=1}^{\infty}[\zeta(2 s)-1]=\frac{3}{4}$ and $\sum_{s=1}^{\infty}[\zeta(2 s+1)-1]=\frac{1}{4}$ have been used for that purpose [1].
One application of the fast converging series is the computation of the irrational number sequences used in encryption coding. The seed number would be any $\zeta(s), s \in \mathbf{R}$. The irrational number sequences can be computed as $\zeta(s+2)=4 R(s)-R(s+2)$ (Lemma 4). This would yield an infinite number of irrational number sequences for encryption keys. The fast recursion for computation of the zeta function maps is also useful in the analysis and synthesis of the log-sampled signals applied in compressive sampling scheme [7].

Theorem 1 and the corresponding applications (Lemmas 1 - 3) give a new converging series for the Riemann zeta function. To the best of authors' knowledge no previous studies concern on the convergence of the similar series. Theoretically, the series of the form $\sum_{s=1}^{\infty} \frac{\zeta(s)}{s !}$, where $s$ ! denotes the factorial function, would guarantee accelerated convergence. However, to conduct the corresponding results (Lemmas 1 - 4) the convergence of the series (26) or some of its variant should be proved. This offers an interesting subject for future work.

\section{Acknowledgements}

This work was supported by the National Technology Agency of Finland (TEKES).

\section{REFERENCES}

[1] J. M. Borwein, D. M. Bradley and R. E. Crandall, "Computational Strategies for the Riemann Zeta Function," Journal of Computational and Applied Mathematics, Vol. 121, No. 1-2, 2000, pp. 247-296. doi:10.1016/S0377-0427(00)00336-8

[2] R. Apéry, "Irrationalité de $\zeta(2)$ et $\zeta(3)$," Astérisque, Vol. 61,1979, pp. 11-13.

[3] F. Beukers, "A Note on the Irrationality of $\zeta(3)$," Bulletin London Mathematical Society, Vol. 11, No. 3, 1979, pp. 268-272.

[4] E. Grosswald, "Remarks Concerning the Values of the Riemann Zeta Function at Integral, Odd Arguments," Journal of Number Theory, Vol. 4, No. 3, 1972, pp. 225-235. doi:10.1016/0022-314X(72)90049-2

[5] D. Cvijovic and J. Klinowski, "Integral Representations of the Riemann Zeta Function for Odd-Integer Arguments," Journal of Computational and Applied Mathematics, Vol. 142, No. 2, 2002, pp. 435-439. doi:10.1016/S0377-0427(02)00358-8

[6] T. Ito, "On an Integral Representation of Special Values of the Zeta Function at Odd Integers," Journal of the Mathematical Society of Japan, Vol. 58, No. 3, 2006, pp. 681-691. doi:10.2969/jmsj/1156342033

[7] H. Olkkonen and J. T. Olkkonen, "Log-Time Sampling of Signals: Zeta Transform," Open Journal of Discrete Mathematics, Vol. 1, No. 2, 2011, pp. 62-65. doi:10.4236/ojdm.2011.12008 